\title{
Experimentelle Untersuchungen zur Bildgüte konventioneller Film- Folien-Mammographie, digitaler Mammographie mit Speicherfolien in Vergrößerungstechnik und voll digitaler Mammographie in CCD-Technik
}

\begin{abstract}
Zusammenfassung. Ziel: Vergleich der Bildgüte zwischen konventioneller Film-Folien-Kontakt-Mammographie, digitaler Speicherfolien-Mammographie in Vergrößerungstechnik und voll digitaler Mammographie in CCD-Technik. Material und Methoden: Testobjekt war ein RMI-Mammaphantom, von dem Aufnahmen mit einem konventionellen und 4 digitalen bildgebenden Systemen angefertigt wurden. Zusätzlich wurden die Aufnahmen eines der digitalen Systeme kontrastbetont nachbearbeitet und in den Vergleich mit einbezogen. Ergebnisse: Voll digitale Mammographie in CCD-Technik erreicht deutlich bessere Ergebnisse in der Detailerkennbarkeit als Mammographien in Film-Folien-Kontakt-Technik bzw. Mammographien in digitaler Speicherfolien-Technik. Schlussfolgerung: Aufgrund der hier gefundenen Ergebnisse ergibt sich für voll digitale Mammographie in CCD-Technik die Möglichkeit, die konventionelle Film-Folien-Kontakt-Mammographie zu ersetzen - dies müssen weitere Untersuchungen zeigen.
\end{abstract}

Schlüsselwörter: Mammographie - Digitale Mammographie Phantomstudie

Image quality in conventional film screen system, digital phosphor storage plate mammography in magnification technique and digital mammography in CCD-technique. Purpose: Comparison of image quality between conventional film screen system, digital phospor storage plate mammography in magnification technique and digital mammography in CCD-technique. Materials and Methods: Radiograms of a RMImammography phantom were acquired using a conventional film screen system, two digital storage plate systems and two digital systems in CCD-technique. Additionally, the radiograms of one digital phosphor storage plate system were postprocessed emphasizing contrast and included in the comparison. Results: The detectability of details was the best with the digital mammography in CCD-technique in comparison with the conventional film screen technique resp. digital phosphor storage plate in magnification technique. Conclusions: Based on these results there is the possibility to replace the conventional film screen system by further studies - this has to be confirmed.

Key words: Mammography - Digital mammography - Phantom study

Fortschr Röntgenstr 2001; 173: 965-968

(c) Georg Thieme Verlag Stuttgart · New York

ISSN 1438-9029
R. Schulz-Wendtland ${ }^{1}$, U. Aichinger ${ }^{1}$, M. Säbel ${ }^{2}$,

C. Böhner ${ }^{1}$, M. Dobritz ${ }^{1}$, W. Bautz ${ }^{1}$

${ }^{1}$ Institut für Diagnostische Radiologie,

Friedrich-Alexander-Universität Erlangen-Nürnberg

2 Klinik und Poliklinik für Strahlentherapie,

Friedrich-Alexander-Universität Erlangen-Nürnberg

\section{Einleitung}

Seit Februar 1998 wird im Institut für Diagnostische Radiologie, Gynäkologische Radiologie, der Universität Erlangen-Nürnberg die digitale Lumineszenzradiographie mit Speicherfolien in Vergrößerungstechnik (Fa. Fuji) als Routinemethode für die Mammographie eingesetzt $(n=11217)[4,12,13]$.

Experimentelle Untersuchungen zur Detektion und Diagnosestellung bei Mikrokalzifikationen und Herdbefunden haben eine Gleichwertigkeit hinsichtlich der Genauigkeit der Diagnosestellung von konventioneller Film-Folien-Mammographie und digitaler Speicherfolienmammographie in Vergrößerungstechnik ergeben [1].

Seit einem Jahr ist ein voll digitaler small field CCD-Detektor (Fa. Siemens) auf dem Markt, welcher bei der Abbildung eines konventionellen Bleistrichrasters eine Grenzauflösung von $10 \mathrm{Lp} / \mathrm{mm}$ (Pixelgröße $48 \mu \mathrm{m}$ ) bzw. $20 \mathrm{Lp} / \mathrm{mm}$ (Pixelgröße $24 \mu \mathrm{m}$ ) erreicht [14].

Um einen ersten Eindruck von der zu erwartenden Bildqualität zu erhalten, wurde eine Untersuchung mit einem Bildgütetestverfahren durchgeführt, das sich in ähnlicher Ausführung seit vielen Jahren bei der Qualitätssicherung in den USA bewährt hat [6].

\section{Material und Methoden}

Aufnahmeobjekt war ein Wisconsin Mammographic Random Phantom (RMI-Phantom), Modell 152A, der Firma Radiation Measurements Inc. Wisconsin USA [6].

Das Phantom ist aus 16 gegeneinander austauschbaren Wachsquadern aufgebaut, die nebeneinander in jeweils 4 Spalten und 4 Reihen in einem Plexiglasblock eingepasst sind. 5 Quader enthalten Aluminiumoxidkörnchen mit Durchmessern zwischen 200 und $740 \mu \mathrm{m}, 6$ Quader Nylonfäden mit einem Querschnitt zwischen 0,4 und 1,6 mm, 4 Quader unscharf begrenzte „Rundherde“ mit Schichtdicken zwischen 5 und 14 mm, 1 Quader ist „leer“. Die Schichtdicke des Phantoms beträgt $45 \mathrm{~mm}$ und entspricht damit der Schichtdicke des Standardphantoms im „European Protocol on Dosimetry in Mammography“ [15]. 
Tab. 1 Technische Spezifikationen der 6 verschiedenen Untersuchungsmodalitäten.

\begin{tabular}{|c|c|c|c|c|c|c|c|}
\hline Technik & Gerät & Hersteller & Anode & Filter & kV & Raster & Vergrößerung \\
\hline Film-Folien-Technik & Mammomat 3000 & Siemens AG & Mo & $30 \mu \mathrm{mMo}$ & 28 & ja & nein \\
\hline Speicherfolientechnik 1 & Mammomat 3 & Siemens AG & Mo & $25 \mu \mathrm{mRh}$ & 35 & nein & 1,8 \\
\hline Speicherfolientechnik 2 & DIMA Plus M 11 & Feinfocus & W & $25 \mu \mathrm{mRh}$ & 35 & nein & 1,7 \\
\hline Speicherfolientechnik 3 & DIMA Plus M 11 & Feinfocus & W & $25 \mu \mathrm{mRh}$ & 35 & nein & 1,7 \\
\hline Opdima 1 & Mammomat 3000 & Siemens AG & Mo & $30 \mu \mathrm{mMo}$ & 28 & ja & nein \\
\hline Opdima 2 & Mammomat 3000 & Siemens AG & Mo & $30 \mu \mathrm{mMo}$ & 28 & ja & nein \\
\hline
\end{tabular}

Die begleitenden Dosismessungen wurden mit diesem Standardphantom und der in der Literatur $[7,15]$ näher beschriebenen Methodik durchgeführt. Die Strahlenexpositionen wurden dabei als Dosisgröße die Luftkerma auf der Strahleneintrittsseite des Phantoms gemessen (siehe auch Tab. 2).

Nach zuvor durchgeführten orientierenden Dosismessungen wurden 6 verschiedene Untersuchungsmodalitäten verglichen (siehe auch Tab.1):

1. Film-Folien-Technik (FF): Die Rasteraufnahmen wurden an einem Mammomat 3000 (Siemens AG) mit der Molybdänanode unter Verwendung eines $30 \mu \mathrm{m}$ Molybdän-Zusatzfilters bei einer Röhrenspannung von $28 \mathrm{kV}$ angefertigt (Auflösung $12 \mathrm{Lp} / \mathrm{mm}$ ). Belichtet wurde die Film-FolienKombination UM Mammo Fine (Folie) UM-MA (Film) der Firma Fuji. Die Entwicklung der Filme erfolgte im Tageslichtentwicklungssystem MF FPM 2100 (Fuji).

2. Speicherfolientechnik 1 (SF 1): Die Aufnahmen wurden am Mammomat 3 (Siemens AG) mit der Molybdän-Anode unter Verwendung eines $25 \mu \mathrm{m}$ Rhodium-Filters, ohne Raster und mit einem Vergrößerungsfaktor von 1,8 bei einer Röhrenspannung von $35 \mathrm{kV}$ belichtet (Auflösung $8 \mathrm{Lp} / \mathrm{mm}$ ). Speicherfolie war der Typ IPHRV (Fuji). Die belichteten Speicherfolien wurden im Image Reader FRC AC 3 M (Fuji) ausgelesen, an den Laserprinter FL-IM (Fuji) geschickt und auf dem Film CR 780-H (FUJI) als Hardcopies ausgedruckt.

3. Speicherfolientechnik 2 (SF 2): Angefertigt wurden die Aufnahmen an der Röntgeneinrichtung DIMA Plus M 11 (Feinfocus) mit der Wolfram-Anode, einem $25 \mu \mathrm{m}$ RhodiumFilter und mit einem Vergrößerungsfaktor von 1,7 ohne Raster bei einer Röhrenspannung von 35 kV (Auflösung 8 Lp/ $\mathrm{mm}$ ). Die Bildverarbeitung war der unter Speicherfolientechnik 1 beschriebenen identisch.

4. Speicherfolientechnik 3 (SF 3): Hier wurden die mit Speicherfolientechnik 2 erstellten digitalen Bilder der Workstation HI-C654 (Fuji) kontrastbetont nachbearbeitet (Auflösung $8 \mathrm{Lp} / \mathrm{mm}$ ). Die Erstellung der Hardcopies erfolgte wie oben beschrieben.

5. Voll digitale Technik 1 (CCD 1): Angefertigt wurden die Aufnahmen mit einem Mammomat 3000 (Siemens AG) mit

Tab. 2 Luftkerma auf der Strahleneintrittsseite des Standardphantoms nach [15] für die 6 verschiedenen Untersuchungsmodalitäten.

\begin{tabular}{lc}
\hline Untersuchungsmodalität & Luftkerma in mGy \\
\hline Film-Folien-Technik (FF) & 9,64 \\
Speicherfolientechnik 1 (SF 1) & 9,54 \\
Speicherfolientechnik 2 (SF 2) & 6,22 \\
Speicherfolientechnik 3 (SF 3) & 6,22 \\
CCD 1 (48 $\mu$ m Pixel) & 10,50 \\
CCD 2 (24 $\mu$ m Pixel) & 13,40 \\
\hline
\end{tabular}

einer Molybdän-Anode unter Verwendung eines $30 \mathrm{~mm}$ Molybdän-Zusatzfilters, einer Röhrenspannung von $28 \mathrm{kV}$ in Kombination mit einem voll digitalen CCD-Detektor (Pixelgröße $48 \mu \mathrm{m}$ ) (Auflösung $10 \mathrm{Lp} / \mathrm{mm}$ ). Die Filmverarbeitung sowie die Erstellung der Hardcopies erfolgten am Dry View TM 8600 Laser Imaging System for digital Mammography (Kodak) mit einer Auflösung von 12,9 Lp/ $\mathrm{mm}$.

6. Voll digitale Technik 2 (CCD 2): Gleiche Untersuchungsmodalitäten wie bei voll digitaler Technik 1 (CCD 1), jedoch mit einem voll digitalen CCD-Detektor und einer Auflösung von $24 \mu \mathrm{m}$ Pixelgröße $(20 \mathrm{Lp} / \mathrm{mm})$. Die Bildverarbeitung sowie Erstellung der Hardcopies erfolgten in gleicher Weise wie bei voll digitaler Technik 1 (CCD 1).

Um Lerneffekte (sogenannte „Reading Order Effects“) bei den Untersuchern zu vermeiden, wurden pro Untersuchungsmodalität je drei verschiedene Phantomkonfigurationen aufgenommen. Alle Bilder wiesen eine optische Nettodichte von etwa 1,3 auf. Die 18 Aufnahmen wurden durch Nummern kodiert und in willkürlicher Reihenfolge 5 Untersuchern zur Auswertung vorgelegt. Die Untersuchergruppe bestand aus 4 Radiologen mit langjähriger Mammographieerfahrung und einem mit der Auswertung von Phantomaufnahmen vertrauten Medizinphysiker. Die Analyse der Bilder erfolgte unter standardisierten Bedingungen für alle Untersucher an demselben Lichtkasten. Die Zuhilfenahme einer Lupe war erlaubt. Die Betrachtungszeit pro Aufnahme war auf 5 Minuten begrenzt. Die von den Untersuchern gemachten Beobachtungen wurden für jede Aufnahme gesondert protokolliert, wobei die einzelnen Befunde für jeden der 16 Quader mit „K“ für Körnchen, „R“ für Rundherd, „F“ für Fäden und „L“ für leer abgekürzt wurden. Die Ergebnisse der Untersucher wurden mit der tatsächlichen Anordnung der Details verglichen, wobei der Test zur Erkennung nur richtig positiver Ergebnisse konzipiert war, d.h. die Sensitivität der 6 Systeme ermittelt wurde. Dementsprechend waren für jede Untersuchungsmodalität maximal 18 Fäden, 15 Körnchen und 12 Rundherde erkennbar, also maximal 45 richtig positive Ergebnisse möglich.

\section{Ergebnisse}

In Tab. 3 sind die Ergebnisse der Auswertung dargestellt, aufgeschlüsselt nach Untersuchungsmodalität, Detailtyp und Untersucher. Außerdem wurden entsprechende Summen gebildet. Diese Summen (S) und die Mittelwerte (MW) über alle Details und Untersucher (FF: S191/MW 38,2, SF1: S190/MW 38,0, SF2: S182/MW 36,4, SF 3: S188/MW 37,6, CCD1: S214/MW 42,8, CCD2: S213/MW 42,6) zeigen, dass sich die 2 Untersuchungsmodalitäten (CCD1 und CCD2) deutlich in der Detailerkennbarkeit von den vier anderen Untersuchungsmodalitäten (FF, SF1, SF2, SF3) unterscheiden. Am schlechtesten 
schneidet SF2 ab, die Modalität mit der geringsten Dosis (siehe auch Tab. 2). Die Nachbearbeitung (SF3) führte bei diesen Bildern dann zu nahezu identischen Ergebnissen wie FFS und SF2.

\section{Diskussion}

In der konventionellen Film-Folien-Mammographie wird die Qualität des Abbildungssystems anhand des Auflösungsvermögens, angegeben als Erkennbarkeitsgrenze der Ortsfrequenz in Linienpaaren pro $\mathrm{mm}(\mathrm{Lp} / \mathrm{mm})$, ermittelt. Die derzeit zugelassenen oder teilzugelassenen Systeme der digitalen Vollfeldmammographie sind limitiert durch die geringere Ortsauflösung $[3,9]$. So erreicht die digitale Mammographie bei Röntgenaufnahmen eines Bleistrichrasters mit Speicherfolien in Kontakttechnik eine Ortsauflösung von $5 \mathrm{Lp} / \mathrm{mm}$, in Vergrößerungstechnik $8 \mathrm{Lp} / \mathrm{mm}$, andere Systeme (z.B. Senograph $2000 \mathrm{D}$, Fa. GE) $5 \mathrm{Lp} / \mathrm{mm}$. Gegenstand der derzeitigen Diskussion ist der Einsatz voll digitaler MammographieSysteme mit einer geringeren Auflösung ( $5 \mathrm{Lp} / \mathrm{mm}$ entsprechend $100 \mu \mathrm{m}$ Pixel) als gemäß der Abnahmeprüfung nach $\S 16$ der Röntgenverordnung gefordert [10]. Mehrere Untersuchergruppen $[4,5,8]$ konnten zeigen, dass die Detailerkennbarkeit in Phantomuntersuchungen trotz geringerer Ortsauflösung gleichwertig ist, und auch in den bisher publizierten klinischen Studien stellte diese für die diagnostische Aussagekraft kein Problem dar $[1,2,11]$.

Die vorliegenden Ergebnisse zeigen jedoch, dass in der voll digitalen Mammographie mit CCD-Detektoren (CCD 1 und CCD 2) und einer Ortsauflösung von $10 \mathrm{Lp} / \mathrm{mm}$ bzw. $20 \mathrm{Lp} /$ mm eine signifikant ( $\mathrm{p}<0,001, \mathrm{Chi}^{2}$-Test) bessere Detailerkennbarkeit zu erzielen ist als in der digitalen Mammographie mit Speicherfolien in Vergrößerungstechnik (Faktor 1,8 bzw. 1,7) sowie der konventionellen Film-Folien-Mammographie in
Kontakt-Technik (siehe auch Tab.1 und 3). Diese bessere Detailerkennbarkeit ist sicherlich zum einen auf die hohe Ortsauflösung der CCD-Systeme zurückzuführen. Die Überlegenheit gegenüber dem Film-Folien-System mit ähnlich hoher Auflösung zeigt, dass die bessere Modulationsübertragungsfunktion des CCD-Systems für den Kontrast wohl der entscheidende Faktor ist. Die beiden CCD-Systeme erreichen vergleichbare Ergebnisse, wobei die hohe Auflösung des CCD 2 limitiert ist durch die maximale Auflösung des Laserprinters von $12,9 \mathrm{Lp} / \mathrm{mm}$, so dass das CCD 2-System auch in der Hardcopy nur 12,9 Lp/mm erreichen kann. Es bleibt abzuwarten, ob durch die Befundung an einem hochauflösenden Monitor noch bessere Ergebnisse erzielt werden können.

Unsere Ergebnisse zeigen ferner, dass eine Steigerung der Detailerkennbarkeit bei Bildern mit reduzierter Dosis (SF2) durch die Nachbearbeitung (SF3) erreicht werden kann. Falls diese Ergebnisse auf klinische Mammographien übertragbar sind, wäre eine Dosisreduktion in der digitalen Mammographie im Vergleich zu den Film-Folien-Systemen zu erwarten $[4,5,10]$. Die CCD-Technik mit einer Ortsauflösung von $10 \mathrm{Lp} /$ $\mathrm{mm}$ hat im Vergleich zum FFS einen gleich hohen Dosisbedarf. Die CCD 2-Technik zeigt keine besseren Ergebnisse als CCD 1 trotz der höheren Dosis, die aber noch unter dem von der Europäischen Kommission festgelegten Grenzwert von 14 mGy liegt [15].

Die Problematik der CCD-Detektoren sind die Schwierigkeiten der Herstellung von Vollfeld-Systemen, so dass zur Zeit nur ein System realisiert ist (Fa. Trex). Die von E. Pisano et al. [9] vorgestellte Studie weist aber nach, dass dieses System mit den Vorteilen der digitalen Systeme und gleichzeitig hoher Auflösung eine Überlegenheit im klinischen Alltag darstellt. Unsere Phantomstudie zeigt ebenfalls, dass ein Mehr an Auflösung (CCD 1 und 2) wohl zu besseren Ergebnissen führt,

\begin{tabular}{|c|c|c|c|c|c|c|c|c|}
\hline \multirow[t]{2}{*}{ Modalität } & & \multirow[t]{2}{*}{$\max$. } & \multicolumn{5}{|c|}{ Untersucher } & \multirow{2}{*}{$\begin{array}{l}\text { Summe } \\
\text { (225 max. } \\
\text { möglich) }\end{array}$} \\
\hline & & & A & B & $\mathrm{C}$ & $\mathrm{D}$ & E & \\
\hline \multirow[t]{4}{*}{ FF } & Fäden & 18 & 13 & 12 & 13 & 12 & 14 & \\
\hline & Körnchen & 15 & 15 & 12 & 15 & 15 & 14 & \\
\hline & Rundherde & 12 & 12 & 9 & 12 & 11 & 12 & \\
\hline & Summe & 45 & 40 & 33 & 40 & 38 & 40 & 191 \\
\hline \multirow[t]{4}{*}{ SF 1} & Fäden & 18 & 13 & 12 & 12 & 12 & 14 & \\
\hline & Körnchen & 15 & 15 & 14 & 15 & 15 & 14 & \\
\hline & Rundherde & 12 & 12 & 9 & 11 & 11 & 11 & \\
\hline & Summe & 45 & 40 & 35 & 38 & 38 & 39 & 190 \\
\hline \multirow[t]{4}{*}{ SF 2} & Fäden & 18 & 12 & 10 & 13 & 12 & 13 & \\
\hline & Körnchen & 15 & 15 & 13 & 14 & 14 & 15 & \\
\hline & Rundherde & 12 & 11 & 8 & 11 & 10 & 11 & \\
\hline & Summe & 45 & 38 & 31 & 38 & 36 & 39 & 182 \\
\hline \multirow[t]{4}{*}{ SF 3} & Fäden & 18 & 12 & 12 & 12 & 13 & 13 & \\
\hline & Körnchen & 15 & 14 & 13 & 15 & 15 & 15 & \\
\hline & Rundherde & 12 & 11 & 9 & 12 & 11 & 11 & \\
\hline & Summe & 45 & 37 & 34 & 39 & 39 & 39 & 188 \\
\hline \multirow[t]{4}{*}{ CCD1 $48 \mu$} & Fäden & 18 & 17 & 12 & 17 & 18 & 18 & \\
\hline & Körnchen & 15 & 15 & 12 & 15 & 15 & 15 & \\
\hline & Rundherde & 12 & 12 & 12 & 12 & 12 & 12 & \\
\hline & Summe & 45 & 44 & 36 & 44 & 45 & 45 & 214 \\
\hline \multirow[t]{4}{*}{$\operatorname{CCD} 224 \mu$} & Fäden & 18 & 18 & 12 & 17 & 18 & 17 & \\
\hline & Körnchen & 15 & 15 & 11 & 15 & 15 & 15 & \\
\hline & Rundherde & 12 & 12 & 12 & 12 & 12 & 12 & \\
\hline & Summe & 45 & 45 & 35 & 44 & 45 & 44 & 213 \\
\hline
\end{tabular}

Tab. 3 Darstellung der richtig positiven Ergebnisse der 5 Untersucher (A-E) zu den 6 Untersuchungsmodalitäten ( $\mathrm{FF}=$ Film-FolienSystem, SF 1 - 3 = Speicherfolientechnik 1 $3, C C D 1$ und $C C D 2=$ voll digitale Technik 1 und 2) aufgeschlüsselt nach Detailgruppen, jeweils der maximal möglichen Anzahl gegenübergestellt (max.). Die letzte Spalte zeigt die Summe der Ergebnisse der 5 Untersucher. 
und zwar in diesem Fall auch deutlich besser als mit dem konventionellen Film-Folien-System. Lassen sich diese Ergebnisse auf klinische Mammographien übertragen und stehen solche hochauflösenden Systeme für die digitale VollfeldMammographie in Zukunft zur Verfügung, so ist mit einer differenzierteren Mammadiagnostik zu rechnen.

\section{Literatur}

${ }^{1}$ Aichinger U, Schulz-Wendtland R, Dobritz M, Mitze M, Bautz W. Mikrokalkanalyse - welche Vorteile zeigt die Speicherfolientechnik gegenüber konventionellen Film-Folien-Systemen? RöFo 1999; 170: 129

${ }^{2}$ Cowen AR, Launders JH, Jadav M, Brettle DS. Visibility of microcalcifications in computed and screen-mammography. Med Phys Biol 1997; 42: 1533-1548

${ }^{3}$ Feig SA, Yaffe MJ. Digital Mammography. Radio Graphics 1998; 18 : $893-901$

${ }^{4}$ Fiedler E, Aichinger U, Böhner C, Säbel M, Schulz-Wendtland R, Bautz W. Bildgüte und Strahlenexposition bei der digitalen Mammographie mit Speicherfolien in Vergrößerungstechnik. RöFo 1999; 171: 60-64

${ }^{5}$ Funke M, Hermann KP, Breiter N, Hundertmark C, Sachs J, Gruhl T, Sperner W, Grabbe E. Digitale Speicherfolienmammographie in Vergrößerungstechnik: Experimentelle Untersuchungen zur Ortsauflösung und zur Erkennbarkeit von Mikrokalk. Fortschr Röntgenstr 1997; 167: 174-179

${ }^{6}$ Kimme-Smith C, Bassett LW, Gold RH. A review of mammography test objects for the calibration of resolution, contrast and exposure. Med Phys 1989; 16: 758-765

${ }^{7}$ Klein R, Aichinger H, Dierker J, Jansen MTJ, Joite-Barfuß S, Säbel M, Schulz-Wendtland $\mathrm{R}$, Zoetelief J. Determination of average glandular dose with modern mammography units for two large groups of patients. Phys Med Biol 1997; 42: 651 -671

8 Obenauer S, Hermann KP, Schorn C, Funke M, Fischer U, Grabbe E. Digitale Vollfeldmammographie: Phantomstudie zur Detektion von Mikrokalk. Fortschr Röntgenstr 2000; 172: 646-650

${ }^{9}$ Pisano ED, Yaffe MJ, Hemminger BM, Hendrick RE, Niklason LT. Current Status of Full-Field Digital Mammography. Acad Radiol 2000; 7: $266-280$

10 Säbel M, Aichinger U, Schulz-Wendtland R, Bautz W. Digitale Vollfeld-Mammographie: Physikalische Grundlagen und klinische Aspekte. Röntgenpraxis 1999; 52: 171 - 177

${ }^{11}$ Schönhofen H, Arnold W, Hess T, Allgayer B. Digitale Mammographie: Erfahrungen bei klinischer Anwendung. Fortschr Röntgenstr 1998; 169: $45-52$

12 Schulz-Wendtland R, Aichinger U, Fiedler E, Böhner C, Säbel M, Bautz W. Digitale Mammographie mit Speicherfolientechnik im täglichen klinischen Einsatz ( $\mathrm{n}=5.150$ ). RöFo 1999; 170: 128 - 129

${ }^{13}$ Schulz-Wendtland R, Aichinger U, Fiedler E, Säbel M, Bautz W. Digital luminescence mammography in clinical use $(n=4.500)$. BJR 1999; 72: 94

${ }^{14}$ Thunberg S, Sklebitz H, Ekdahl B, Bätz L, Lundin A, Möller H, Fleischmann F, Kreider G, Weidner T. OPDIMA: Large - area CCD - based X-ray image sensor for spot imaging and biopsy control in mammography. SPIE 1999; 3659: 150-158

15 Zoetelief J, Fitzgerald M, Leitz W, Säbel M. European protocol on dosimetry in mammography. EUR 16263 Brüssel. European Commission 1996
Prof. Dr. med. R. Schulz-Wendtland

Universität Erlangen-Nürnberg

Institut für Diagnostische Radiologie

Gynäkologische Radiologie

Universitätsstraße 21 - 23

91054 Erlangen 